\title{
Taxonomic Studies in Tribe Loteae (Fabaceae) in Egypt. I: Subtribe Anthyllidinae (Anthyllis, Hymenocarpos and Tripodion)
}

\author{
Zaki A. Turki, Faiza A. Shehata\#, Esam M. Aqlan \\ Botany and Microbiology Department, Faculty of Science, Menoufia University, \\ Shebein El-Koom, Egypt.
}

\begin{abstract}
$\mathbf{T}$
HE PLANT morphology, anatomical characters of stem and leaves, SEM of seed coat surfaces were used to reassess the conflicted taxonomic relationships between the genera Anthyllis, Hymenocarpos and Tripodion. Previous studies treated the genera Hymenocarpos and Tripodion as synonyms to Anthyllis. The present study clearly indicated considerably differences between the three genera. The most important characters used to distinguish the studied genera are habit, inflorescence type, bract shape, calyx, pod shape and seed coat pattern. On the bases of morphology, anatomy and seed characters, three distinct genera, Anthyllis, Hymenocarpos and Tripodion are represented in the Egyptian flora, each with only one species.
\end{abstract}

Keywords: Anthyllis, Hymenocarpos, Tripodion, Morphological, Anatomy, SEM seed.

\section{Introduction}

The genus Anthyllis L. and Hymenocarpos Savi. are two genera of the family Fabaceae, belonging to the subfamily Faboideae Rudd., tribe Loteae DC., subtribe Anthyllidinae W.D.J. Koch. The two genera distributed in Mediterranean regions, with some taxa extending into northern Europe, the Atlantic islands, Northern and Eastern Africa, and Western Asia (Polhill, 1981; Castroviejo, 2000 and Sokoloff, 2003 a).

Tournefort (1719) was the first who gave a more or less exact vision of the genus Anthyllis (syn. Vulneraria), recognizing five species. Linnaeus (1753) included 10 species under the genus Anthyllis, of which A. tetraphylla and $A$. vulneraria, and treated Hymenocarpos circinnatus as Medicago circinnata. Medikus (1787) separated Anthyllis tetraphylla as genus Tripodion, including one species; T. tetraphylla.Savi (1798) separated Medicago circinnata as genus Hymenocarpos, including one species; $H$. circinnatus. He also reported Anthyllis with two species; A. tetraphylla and $A$. vulneraria.

Moench (1794) and Gussone (1827/28) included Anthyllis tetraphylla as synonymy to Vulneraria tetraphylla. Boissier (1838, 1839-1845) separated Anthyllis tetraphylla as genus Physanthyllis, including one species; $P$. tetraphylla. Boissier (1872) recognized Anthyllis into A. vulneraria. $H$. circinnatus, $H$. nummularius and P. tetraphylla. Taubert (1894) included
Hymenocarpos as synonymy to Circinus. Lassen $(1986,1987)$ has shown that Tripodion is an earlier name for Physanthyllis and recognized three species in Tripodion. Akulova $(1985,1986)$ and Sokoloff (2003 a, b) included Hymenocarpos as synonymy to Anthyllis.

In Egypt, Täckholm (1974) recognized genus Anthyllis into A. tetraphylla and A. vulneraria and genus Hymenocarpos into $H$. circinnatus and $H$. nummularius. El-Hadidi \& Fayed (1994/95) and Boulos (1995) recognized Tripodion tetraphyllum as a separate genus and treated $A$. tetraphylla as a synonym. Boulos $(1999,2009)$ treated Tripodion tetraphyllum as a synonym to A. tetraphylla.

The present study aimed to revise critically the taxonomic relationships between the species of the related genera; Anthyllis, Hymenocarpos and Tripodion in Egypt.

\section{Materials and Methods}

The present study is based on available fresh material collected from their natural habitats in Egypt in addition to collections kept in Menoufia University Herbarium (MNF). Seeds of A. vulneraria and Tripodion tetraphyllum are obtained from Institute of Plant Genetics and Crop Research (IPK), Western Regional Plant Introduction Station- Washington State University and Desert Legume Program- The University of Arizona (Table 1). The seeds were cultivated in plastic house, till fruiting stage.

"Corresponding author email: faizashehata@yahoo.com DOI: 10.21608/ejbo.2019.5705.1234

Edited by: Prof. Dr. Fco Martin Huerta-Martinez, University of Guadalajara, Mexico (C)2019 National Information and Documentation Center (NIDOC) 
TABLE 1. Plant names, collection details and sources of seeds included in the present study.

\begin{tabular}{|c|c|c|c|c|c|}
\hline Taxa & $\begin{array}{c}\text { Source of } \\
\text { seeds }\end{array}$ & & Localities & $\begin{array}{c}\text { Geographical } \\
\text { coordinates }\end{array}$ & Date of collection \\
\hline A. vulneraria & PI 311348 & & - & - & - \\
\hline $\begin{array}{l}\text { Hymenocarpos } \\
\text { circinnatus }\end{array}$ & - & $\begin{array}{l}\text { Al-Mathani } \\
\text { Matrouh. } \\
\text { Wadi Umm } \\
\text { Matrouh. }\end{array}$ & $\begin{array}{l}\text { Al-Bahria - Marsa } \\
\text { El-Rakham - Marsa }\end{array}$ & $\begin{array}{l}31^{\circ} 27.963 \backslash \mathrm{N} \\
26^{\circ} 45.287 \backslash \mathrm{E} \\
31^{\circ} 24.076 \backslash \mathrm{N} \\
27^{\circ} 01.704 \mathrm{E}\end{array}$ & 3,4 / 2014-2018 \\
\hline Tripodion tetraphyllum & $\begin{array}{c}\text { Akz-Nr: } \\
\text { ANTHY } 14 \\
\text { (IPK) }\end{array}$ & & - & - & - \\
\hline
\end{tabular}

Samples for anatomy of the stem and mature leaves were chosen from fresh materials. All assessment was made on all plants at similar developmental stages (fruiting stage) and in comparable positions of each plant. Fresh material was fixed in F.A.A. (5:5:90). After fixation stems and leaves were transformed in ethyl alcohol series and then embedded in paraffin wax. The stems and leaves were sectioned at 10$15 \mu \mathrm{m}$; sections were dehydrated in alcohol-xylol series. Sections were stained in safranin and light green according to Sass (1961). The transverse sections were examined and photographed by Zeiss research microscope. A planimeter was used for estimation of the width of each tissue in the section. Terminology followed Abd El-Rahman et al. (1976), Pandey (1982) and Abd El-Gawad et al. (1989).

SEM study of the investigated seeds was carried out by mounting mature seeds on brass stubs and coated with a thin layer of gold using JEOL JSM 530P SEM at the electron microscopic unit, Faculty of Science, Alexandria University. Terminology followed Lersten (1981), Brochmann (1992), Stearn (1992) and Kirkbride et al. (2003).

\section{Results}

Macromorphological studies (Table 2 and Fig. 1)

Anthyllis vulneraria L., Sp. P1. 719 (1753). subsp. maura (Beck) Maire, Bull. Soc. Hist. Nat. Afr. Nord 20: 20 (1929).

Syn. Anthyllis maura Beck, Ann. K. K. Naturhist. Hofmus. 11: 64 (1896).

Perennial herb, $35-45 \mathrm{~cm}$ height, stem decumbent-erect, branched at base, tomentose, cylindrical, whitish green, internodes up to $1 \mathrm{~mm}$ long in decumbent stem while $3-3.5 \mathrm{~cm}$ long in erect branches. Lower leaves simple, while upper leaves imparipinnate, all leaves alternate, whitish green, petiolate or sessile; petiolate leaves with petioles $1.7-3.7 \mathrm{~cm}$ long, puberulent; lamina in simple leaf $4.2-4.5 \times 0.8-0.9 \mathrm{~cm}$, narrow elliptic, acuminate apex, entire margin, glabrous in upper surface and tomentulose in lower surface; imparipinnate leaves opposite-alternate with 3-9 leaflets, petiolules $0.5-1 \mathrm{~mm}$ long; lamina in terminal leaflets $3-5.5 \times 0.9-1.5 \mathrm{~cm}$ while in lateral leaflets $0.5-3 \times 0.3-0.7 \mathrm{~cm}$, lateral leaflets unequal, narrow elliptic-elliptic acuminate-acute apex, entire margin, glabrous in upper surface and tomentose in lower surface; leaf rachis (4-) 14$25 \mathrm{~mm}$ long, stipules filiform. Inflorescence head, 11-23 flowers; peduncle cylindrical, tomentulose, $10-12 \mathrm{~cm}$ long. Bracts palmatisect, $11-17 \mathrm{~mm}$ long, whitish green, pubescent in upper surface but tomentose in lower surface; lower bracts 5-7 lobes while upper bracts 3 lobes, lobes ellipticlanceolate-oblong, 5-13 $\times 2-4 \mathrm{~mm}$, acute apex, entire margin. Flowers $14.5-15 \mathrm{~mm}$ long, pedicel $0.5-1 \mathrm{~mm}$ long, tomentose; calyx tubular, with oblique mouth, white with violet at apex, tube $7-9 \mathrm{~mm}$ long, tomentose in outer surface, teeth lanceolate-triangular, 1-2.5 mm long, unequal, acute apex, entire margin, tomentose in outer surface; standard purple white, lamina 13-14 $\times$ 4.5-5mm, auriculate, entire margin, obtuse apex, claw 6-6.5 mm long, veins violet, 2-3mm long, glabrous; wings purple greenish white, lamina 12$13 \times 2-2.5 \mathrm{~mm}$, ovate, entire margin, rounded apex, claw 8-8.5 mm long, auriculate 0.3-0.5 mm long, truncate apex; keel greenish white, lamina 12-13 $\times$ $1.5-2 \mathrm{~mm}$, straight apex, beak obtuse, dark violet, claw 8-8.5 mm long. Androecium monadelphous, the united filament with the free parts $2-2.5 \mathrm{~mm}$ long, united part 9-10mm long, filament dilated above, anther uniform. Gynoecium stipitate, 
9.5-12.5mm long, gynophore $1.5-2.5 \mathrm{~mm}$ long, glabrous; ovary $2 \times 1 \mathrm{~mm}$, ellipsoid, glabrous, style $7-8 \mathrm{~mm}$ long, lower part thin and purple; stigma capitates. Pod ellipsoid, $4-5 \times 2.5-3 \mathrm{~mm}$, pale brown-creamy, reticulate venation, included within the calyx, beak $0.5-1 \mathrm{~mm}$ long, seeds $1-2$. Seeds ovoid with rounded poles, $2-3 \times 1-1.5 \mathrm{~mm}$, brown or yellowish with terminal part pale green, hilum subapical.

TABLE 2. Morphological characters of the studied taxa.

\begin{tabular}{|c|c|c|c|c|}
\hline Character & & Anthyllis vulneraria & $\begin{array}{l}\text { Hymenocarpos } \\
\text { circinnatus }\end{array}$ & Tripodion tetraphyllum \\
\hline \multirow{5}{*}{ Plant } & \multirow{3}{*}{ Habit } & Herb & Herb & Herb \\
\hline & & Perennial & Annual & Annual \\
\hline & & Decumbent-erect & Decumbent & Decumbent \\
\hline & Height (cm) & $35-45$ & $35-60$ & $20-35$ \\
\hline & Colour & Whitish green & Whitish green & Yellowish green \\
\hline \multirow{5}{*}{ Stem } & Shape & Cylindrical & Cylindrical & Cylindrical \\
\hline & Surface & Tomentose & Pilose & Pilose \\
\hline & Colour & Whitish green & Whitish green & Reddish brown \\
\hline & Branched & At base & At base & At base \\
\hline & Internode length $(\mathrm{cm})$ & $\begin{array}{c}\text { Up to } 1 \mathrm{~mm} \text { in decumbent } \\
\text { stem } \\
\text { 3-3.5 in erect stem }\end{array}$ & $3-6$ & $1-2$ \\
\hline \multirow{2}{*}{ Stipule } & Shape & Filiform & Absent & Absent \\
\hline & Length (mm) & Up to 0.5 & - & - \\
\hline \multirow{4}{*}{ Leaf } & First & - & 2 Simple & 2 Simple \\
\hline & $\begin{array}{l}\text { Type } \quad \text { Lower } \\
\text { Upper }\end{array}$ & $\begin{array}{c}\text { Simple } \\
\text { Imparipinnate }\end{array}$ & Imparipinnate & Imparipinnate \\
\hline & Arrangement & Alternate & Alternate & Alternate \\
\hline & Colour & Whitish green & Whitish green & Yellowish green \\
\hline Leaf rachis & Length (mm) & (4-) $14-25$ & $5-15$ & $3-5$ \\
\hline \multirow{5}{*}{$\begin{array}{l}\text { Limb simple } \\
\text { leaf }\end{array}$} & Shape & Narrow elliptic & Obovate & Elliptic \\
\hline & Size $\mathrm{L} \times \mathrm{W}(\mathrm{cm})$ & $4.2-4.5 \times 0.8-0.9$ & $4-5.2 \times 1.4-1.7$ & $1.5-2 \times 0.6-0.7$ \\
\hline & Apex & Acuminate & Acute-rounded & Acute \\
\hline & Margin & Entire & Entire & Entire \\
\hline & Surface & $\begin{array}{l}\text { Glabrous in upper } \\
\text { Tomentulose in lower }\end{array}$ & Pilose in both & Pilose in both \\
\hline \multirow{3}{*}{ Leaflet } & No. & $3-9$ & $2-7$ & $2-5$ \\
\hline & Petiolule & $0.5-1 \mathrm{~mm}$ & Sessile & Up to $0.5 \mathrm{~mm}$ \\
\hline & & Opposite-alternate & Opposite-alternate & Alternate \\
\hline \multirow{5}{*}{ Limb leaflet } & Shape & Narrow elliptic-elliptic & Elliptic-obovate & Elliptic-obovate \\
\hline & Size $\mathrm{L} \times \mathrm{W}(\mathrm{cm})$ & $\begin{array}{c}\text { Terminal } 3-5.5 \times 0.9-1.5 \\
\text { Lateral } 0.5-3 \times 0.3-0.7\end{array}$ & $\begin{array}{c}\text { Terminal } 3-6 \times 1-2.3 \\
\text { Lateral } 0.7-2.6 \times \\
0.3-1.2\end{array}$ & $\begin{array}{c}\text { Terminal } 1.2-2.5 \times 0.7-1.2 \\
\text { Lateral } 0.3-1.2 \times 0.1-0.6\end{array}$ \\
\hline & Apex & Acuminate-acute & Obtuse-acute & $\begin{array}{l}\text { Terminal rounded-truncate } \\
\text { Lateral acuminate-acute }\end{array}$ \\
\hline & Margin & Entire & Entire & Entire \\
\hline & Surface & $\begin{array}{l}\text { Glabrous in upper } \\
\text { Tomentose in lower }\end{array}$ & Pilose in both & $\begin{array}{l}\text { Pubescent in upper } \\
\text { Tomentulose in lower }\end{array}$ \\
\hline
\end{tabular}


TABLE 2. Cont.

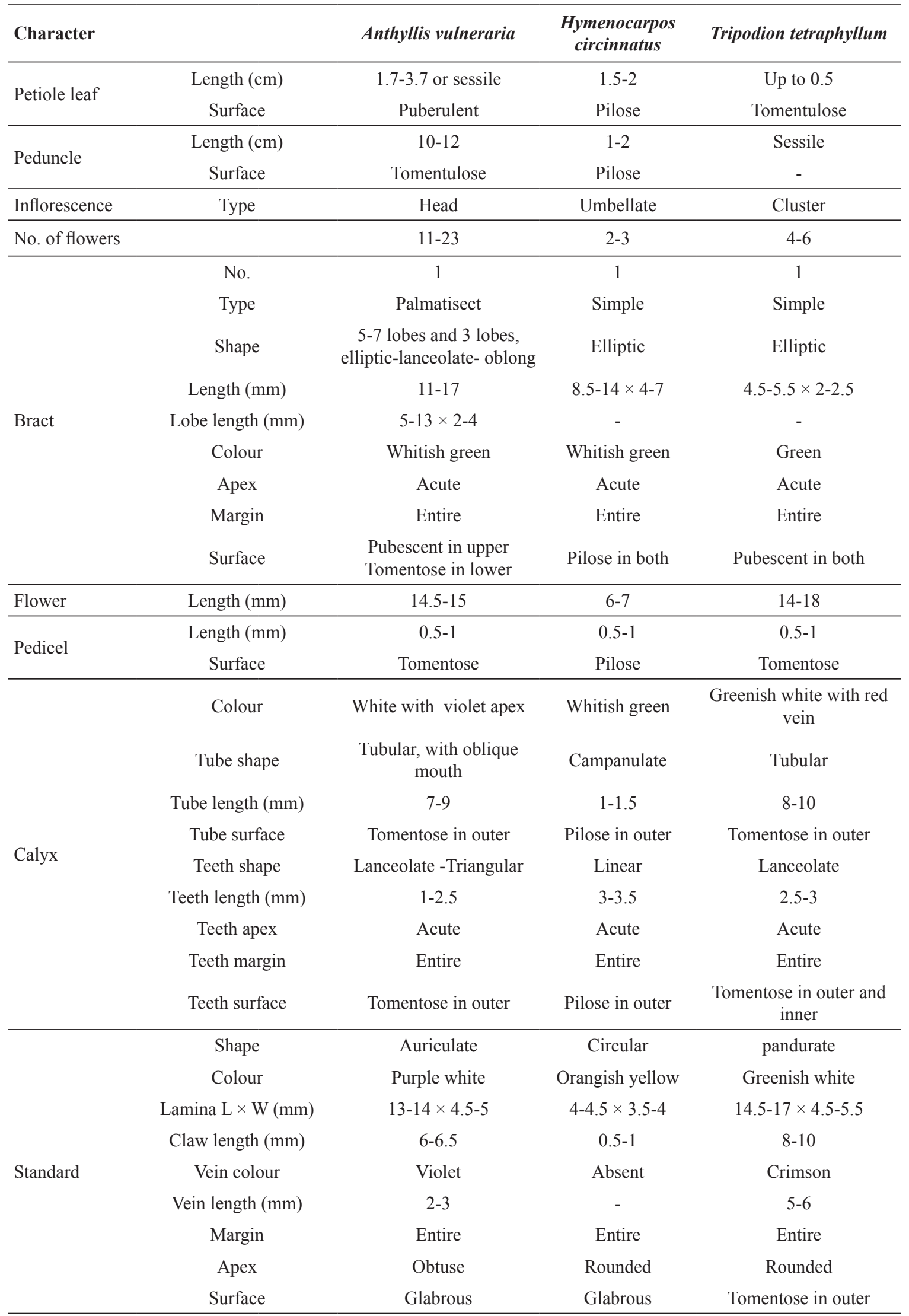


TABLE 2. Cont.

\begin{tabular}{|c|c|c|c|c|}
\hline Character & & Anthyllis vulneraria & $\begin{array}{l}\text { Hymenocarpos } \\
\text { circinnatus }\end{array}$ & Tripodion tetraphyllum \\
\hline \multirow{9}{*}{ Wings } & Shape & Ovate & Oblong & Ovate \\
\hline & Colour & Purple greenish white & Yellow & Greenish yellow \\
\hline & Lamina $\mathrm{L} \times \mathrm{W}(\mathrm{mm})$ & $12-13 \times 2-2.5$ & $4-4.5 \times 1.5-2$ & $12.5-15 \times 2-2.5$ \\
\hline & Claw length (mm) & $8-8.5$ & $1-1.2$ & $8-9$ \\
\hline & Apex & Rounded & Rounded & Rounded \\
\hline & Margin & Entire & Entire & Entire \\
\hline & Surface & Glabrous & Glabrous & Glabrous \\
\hline & Auricule length (mm) & $0.3-0.5$ & $0.4-0.6$ & $0.5-0.8$ \\
\hline & Auricule apex & Truncate & Rounded & Truncate \\
\hline \multirow{6}{*}{ Keel } & Colour & Greenish white & Shiny yellow & Greenish white \\
\hline & Lamina $\mathrm{L} \times \mathrm{W}(\mathrm{mm})$ & $12-13 \times 1.5-2$ & $4.5-6 \times 1.5-2$ & $12-14 \times 1.5-2$ \\
\hline & Claw length (mm) & $8-8.5$ & $1-1.5$ & $9-9.5$ \\
\hline & Apex & Straight beak, obtuse & Straight beak, obtuse & Straight beak, acute \\
\hline & Surface & Glabrous & Glabrous & Glabrous \\
\hline & Beak colour & Dark violet & Yellow & Dark violet \\
\hline Androecium & Type & Monadelphous & Diadelphous & Diadelphous \\
\hline \multirow{5}{*}{ Stamens } & Free length (mm) & - & $3.5-4$ & $10-13$ \\
\hline & $\begin{array}{l}\text { Filaments united (free } \\
\text { parts length } \mathrm{mm} \text { ) }\end{array}$ & $2-2.5$ & $\begin{array}{l}5=3-3.5 \\
4=2-2.5\end{array}$ & $1-2$ \\
\hline & $\begin{array}{l}\text { Filaments united (united } \\
\text { parts length } \mathrm{mm} \text { ) }\end{array}$ & $9-10$ & $2-2.5$ & $9-11$ \\
\hline & & Filament dilated above & $\begin{array}{l}\text { Filament dilated } \\
\text { above }\end{array}$ & Filament dilated above \\
\hline & Anthers & Uniform & Uniform & Uniform \\
\hline \multirow{4}{*}{ Ovary } & Shape & Ellipsoid & Narrow oblong & Narrow oblong \\
\hline & Size $L \times W$ & $2 \times 1$ & $1.2-1.5 \times 0.5$ & $3 \times 0.5$ \\
\hline & Surface & Glabrous & Puberulent & Puberulent in apex \\
\hline & Colour & Green & Green & Light green \\
\hline \multirow{2}{*}{ Gynophores } & Length (mm) & $1.5-2.5$ & $0.2-0.3$ & $1.5-2$ \\
\hline & Surface & Glabrous & Glabrous & Puberulent \\
\hline \multirow{2}{*}{ Style } & Length (mm) & $7-8$ & $4-4.5$ & $6-8$ \\
\hline & & Lower part thin and purple & Lower part thin & Lower part thin \\
\hline Stigma & Shape & Capitates & Ellipsoid & Capitates \\
\hline \multirow{8}{*}{ Pod } & Shape & Ellipsoid & Orbicular- reniform & Ellipsoid - cylindrical \\
\hline & Size L $\times W(m m)$ & $4-5 \times 2.5-3$ & $12-18 \times 11-16$ & $4-5.5 \times 3-3.5$ \\
\hline & Colour & Pale brown-creamy & $\begin{array}{l}\text { Blackish brown-pale } \\
\text { brown-brown }\end{array}$ & Pale brown-creamy \\
\hline & Surface & Reticulate & $\begin{array}{l}\text { Reticulate, appressed } \\
\text { hairy }\end{array}$ & Reticulate, pubescent \\
\hline & Margin & - & $\begin{array}{l}\text { Margins denticulate, } \\
\text { membranous wing }\end{array}$ & - \\
\hline & Constriction & - & - & $\begin{array}{l}\text { Constricted between the } \\
\text { seeds }\end{array}$ \\
\hline & & Included within the calyx & - & Included within the calyx \\
\hline & Beak length (mm) & $0.5-1$ & - & $2-3$ \\
\hline Seed No. & & $1-2$ & 2 & $1-2$ \\
\hline
\end{tabular}



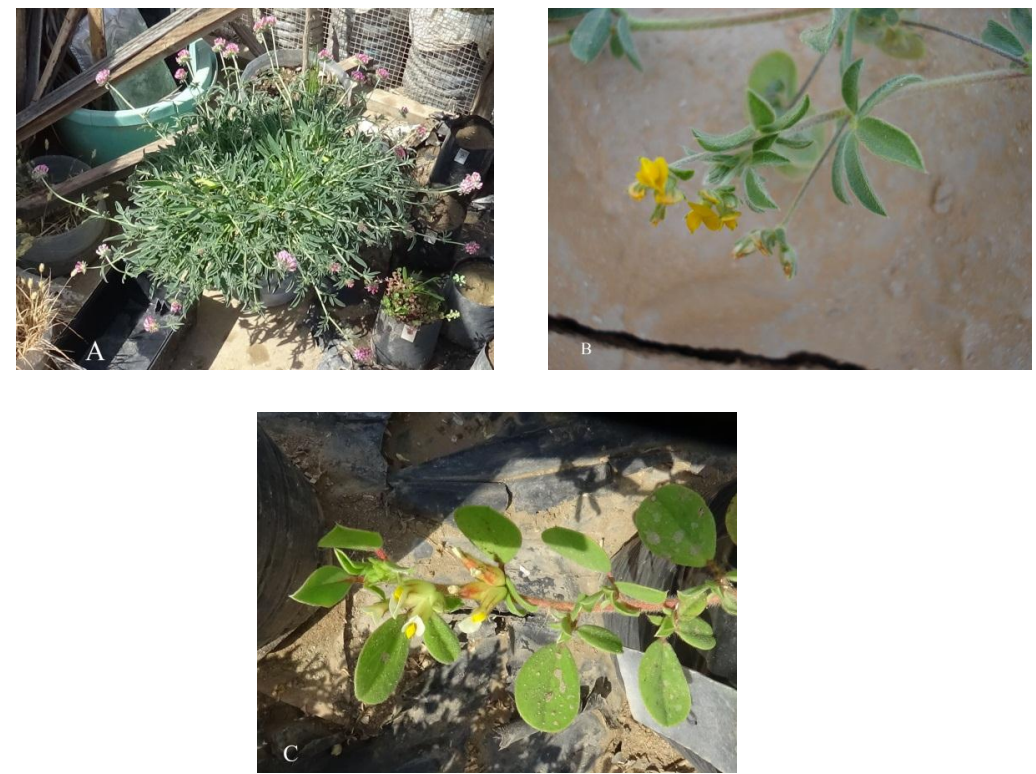

Fig. 1. Habit of the studied taxa: A. Anthyllis vulneraria; B. Hymenocarpos circinnatus; C. Tripodion tetraphyllum.

Hymenocarpos circinnatus (L.) Savi, Fl. Pis. 2:205 (1798).

Syns. Medicago circinnata L., Sp. Pl., ed. 1, 778 (1753). Medicago nummularia DC., Cat. Hort. Monsp. 124 (1813). Hymenocarpos nummularis (DC.) G. Don, Gen. Hist. 2:173 (1832). Hymenocarpos nummularius (DC.) Boiss., Fl. Orient. 2:160 (1872). Cornicina circinnata (L.) Boiss., Voy. Bot. Midi Esp. 2:163 (1839). Anthyllis circinnata (L.) D.D. Sokoloff, Byull. Moskovsk. Obshch. Isp. Prir., 108 (3):46 (2003).

Annual herb, 35-60 cm height, stem decumbent, branched at base, cylindrical, pilose, whitish green, internodes 3-6mm long. The first two leaves simple, alternate, whitish green, petiolate, petioles $1.5-2 \mathrm{~cm}$ long, pilose, lamina $4-5.2 \times 1.4-1.7 \mathrm{~cm}$, obovate, acute-rounded apex, entire margin, pilose in both surfaces; leaves imparipinnate, exstipulate, sessile, with 3-7 leaflets; leaflets opposite-alternate, petiolate- subsessile, elliptic-obovate, obtuseacute apex, entire margin, pilose in both surfaces, unequal; lamina in terminal leaflet 3-6 $\times 1-2.3 \mathrm{~cm}$ while in lateral leaflet $0.7-2.6 \times 0.3-1.2 \mathrm{~cm}$, leaf rachis $5-15 \mathrm{~mm}$ long. Inflorescence umbellate, 2-3 flowers; peduncle 1-2cm long, cylindrical, pilose. Bracts simple, elliptic, 8.5-14 × 4-7mm, whitish green, pilose in both surfaces, acute apex, entire margin. Flowers $6-7 \mathrm{~mm}$ long, pedicel $0.5-1 \mathrm{~mm}$ long, pilose; calyx whitish green, campanulate, tube $1-1.5 \mathrm{~mm}$ long, pilose in outer surface, teeth linear, 3-3.5mm long, equal, acute apex, entire margin, pilose in outer surface; standard orange- yellow, lamina $4-4.5 \times 3.5-4 \mathrm{~mm}$, circular, entire margin, rounded apex, claw veinless, glabrous 0.5-1 mm long; wings yellow, lamina 4-4.5 × 1.5$2 \mathrm{~mm}$, oblong, entire margin, rounded apex, claw $1-1.2 \mathrm{~mm}$ long, auricule $0.4-0.6 \mathrm{~mm}$ long, rounded apex; keel shiny yellow, lamina 4.5-6 × 1.5-2mm, straight apex, beak obtuse, claw 1-1.5mm long. Androecium diadelphous, free filament $3.5-4 \mathrm{~mm}$ long, the other united filament with the free parts have two lengths, five filaments $3-3.5 \mathrm{~mm}$ long, four filaments $2-2.5 \mathrm{~mm}$ long, united part $2-2.5 \mathrm{~mm}$ long, filament dilated above, anther uniform. Gynoecium stipitate, 5.4-6.3mm long, gynophore $0.2-0.3 \mathrm{~mm}$ long, glabrous; ovary 1.2-1.5 $\times 0.5 \mathrm{~mm}$, narrow oblong, puberulent, style $4-4.5 \mathrm{~mm}$ long, lower part thin; stigma ellipsoid. Pod orbicularreniform, 12-18 $\times 11-16 \mathrm{~mm}$, blackish brownpale brown, reticulate venation, appressed hairy, margins denticulate, membranous wing, with two seeds. Seeds reniform, 3-3.5 $\times$ 2-2.5mm, yellowish-creamy-pale brown, with rounded poles, hilum blackish brown and lateral.

Tripodion tetraphyllum (L.) Fourr., Ann. Soc. Linn. Lyon, ser. 2, 16:359 (1868).

Syns. Anthyllis tetraphylla L., Sp. Pl. 719 (1735). Vulneraria tetraphylla (L.) Guss., Fl. Sicul. Prodr. 2:395 (1828-32). Physanthyllis tetraphylla (L.) Boiss., Voy. Bot. Espagne 2:162 (1840).'

Annual herb, 20-35cm height, stem decumbent, branched at base, cylindrical, pilose, reddish brown, internodes $1-2 \mathrm{~mm}$ long. The first two 
leaves simple, alternate, yellowish green, petiolate, petioles up to $0.5 \mathrm{~cm}$ long, tomentulose; lamina 1.5 $2 \times 0.6-0.7 \mathrm{~cm}$, elliptic, acute apex, entire margin, pilose in both surfaces; leaves imparipinnate, exstipulate, alternate, yellowish green, petiolate, petioles up to $0.5 \mathrm{~cm}$ long, tomentulose; leaf $3-5$ leaflets, petiolules up to $0.5 \mathrm{~mm}$ long, lamina in terminal leaflet $1.2-2.5 \times 0.7-1.2 \mathrm{~cm}$ while in lateral leaflet $0.3-1.2 \times 0.1-0.6 \mathrm{~cm}$, leaflets unequal, apex elliptic-obovate, rounded-truncate in terminal leaflet while acuminate-acute in lateral leaflet, entire margin, pubescent in upper surface while tomentulose in lower surface; leaf rachis $3-5 \mathrm{~mm}$ long. Inflorescence axillary clusters, 4-6 flowers. Bracts simple, elliptic, 4.5-5.5 $\times 2-2.5 \mathrm{~mm}$, green, pubescent in both surfaces, acute apex, entire margin. Flowers 14-18mm long, pedicel 0.5-1 $\mathrm{mm}$ long, tomentose; calyx tubular, greenish white with reddish at apex and veined, tube $8-10 \mathrm{~mm}$ long, tomentose in outer surface, teeth lanceolate, 2.5-3mm long, equal, acute apex, entire margin, tomentose in both surfaces; standard greenish white, lamina $14.5-17 \times 4.5-5.5 \mathrm{~mm}$, pandurate, entire margin, rounded apex, claw $8-10 \mathrm{~mm}$ long, veins crimoson, 5-6 $\mathrm{mm}$ long, tomentose in lower surface; wings greenish white, lamina 12.5-15 $\times 2-2.5 \mathrm{~mm}$, ovate, entire margin, rounded apex, claw $8-9 \mathrm{~mm}$ long, auricule $0.5-0.8 \mathrm{~mm}$ long, truncate apex; keel greenish white, lamina 12-14 $\times 1.5-2 \mathrm{~mm}$, straight apex, beak acute, dark violet, claw 9-9.5 mm long. Androecium diadelphous, free filament $10-13 \mathrm{~mm}$ long, the other united filament with the free parts 1-2 mm long, united part 9-11 mm long, filament dilated above, anther uniform. Gynoecium stipitate, $10.5-13 \mathrm{~mm}$ long, gynophore 1.5-2 mm long, puberulent; ovary $3 \times$ $0.5 \mathrm{~mm}$, narrow oblong, puberulent in apex, style 6-8 $\mathrm{mm}$ long, lower part thin; stigma capitate. Pod ellipsoid-cylindrical, 4.5-5 × 3-3.5mm, pale brown-creamy, reticulate venation, pubescent, constricted between the seeds, included within the calyx, beak 2-3mm long, with 1-2 seeds. Seeds oblong-ellipsoid crispate, $3-3.5 \times 2-2.5 \mathrm{~mm}$, brown with black spots, with rounded- truncate poles, hilum lateral.

\section{Anatomical studies}

The anatomical investigations of the stem and leaf of the studied taxa is represented in Tables 3 , 4 and Fig. 2, 3.

\section{Stem anatomy}

Anthyllis vulneraria

Stem circular, 1.2-1.3mm diameter; epidermal layer $12.5-15 \mu \mathrm{m}$ thickness covered with $2.5-5 \mu \mathrm{m}$ cuticle thickness. Cortex 7-8 parenchymatous layers, $100-137.5 \mu \mathrm{m}$ thickenss. Vascular cylinder 15-16 bundles, each bundle 150-200 $\mu \mathrm{m}$ long, pericyclic fibers 3-4 layers, $37.5-55 \mu \mathrm{m}$ thickness. Phloem 3-4 layers, 20-30 $\mu \mathrm{m}$ thickness. Cambium 2 layers, $10-12.5 \mu \mathrm{m}$ thickness. Xylem 2-7 arches, 4-5 vessels per arch, 100-160 $\mu \mathrm{m}$ length. Pith cells parenchymatous, $520-530 \mu \mathrm{m}$ diameter.

\section{Hymenocarpos circinnatus}

Stem circular, 1.9-2mm diameter; epidermal layer $12.5-20 \mu \mathrm{m}$ thickness covered with 2.5-5 $\mu \mathrm{m}$ cuticle thickness. Cortex 5-6 layers parenchymatous; $112.5-175 \mu \mathrm{m}$ thickenss. Vascular cylinder 16-17 bundles, each bundle 220-300 $\mu \mathrm{m}$ long, pericyclic fibers 4-5 layers, $50-75 \mu \mathrm{m}$

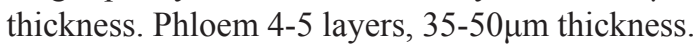
Cambium 2 layers, 10-12.5 $\mu \mathrm{m}$ thickness. Xylem

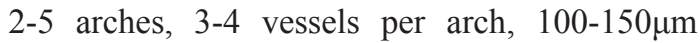
length. Pith cells parenchymatous, $1000-1025 \mu \mathrm{m}$ diameter.

\section{Tripodion tetraphyllum}

Stem circular, $1.9-2.1 \mathrm{~mm}$ diameter; epidermal layer $12.5-22.5 \mu \mathrm{m}$ thickness covered with 2.5-5 $\mu \mathrm{m}$ cuticle thickness. Cortex 7-9 layers parenchymatous; $200-370 \mu \mathrm{m}$ thickenss, with solitary crystals. Vascular cylinder 17-19 bundles, each bundle $140-270 \mu \mathrm{m}$ wide, pericyclic fibers 4-6 layers, 40-62.5 $\mu \mathrm{m}$ thickness. Phloem 3-4 layers, $25-37.5 \mu \mathrm{m}$ thickness. Cambium 2 layers, $12.5-15 \mu \mathrm{m}$ thickness. Xylem 2-8 arches, 3-8 vessels per arch, 60-150 $\mu \mathrm{m}$ length. Pith cells parenchymatous, 950-975 $\mu \mathrm{m}$ diameter, with solitary crystals. Tannin cells few in pith.

\section{Leaf anatomy \\ Anthyllis vulneraria}

The leaf in midrib region is v-shaped, 400$420 \mu \mathrm{m}$ thickness, upper and lower epidermis uniseriate, $12.5-25 \mu \mathrm{m}$ thickness, covered with $2.5 \mu \mathrm{m}$ cuticle thickness. In midrib region, parenchyma 2-3 layers, isodiametric- flattened, 87.5-100 $\mu \mathrm{m}$ thickness. Xylem 4-6 arches, 2-6 vessels per arch, xylem arch 75-82.5 $\mu \mathrm{m}$ length.

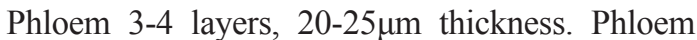
fibers at midrib region below the main vascular bundle, 3-4 layers, 25-37.5 $\mu \mathrm{m}$ thickness. Below the main vascular bundles 3-4 layers of parenchyma cells, $100-107.5 \mu \mathrm{m}$ thickness. The wings 300 $350 \mu \mathrm{m}$ thickness, mesophyll tissue chlorophyllous

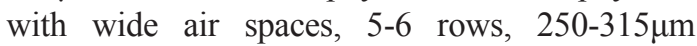
thickness, with solitary crystals. 
TABLE 3. Anatomical characters of stem in the studied taxa.

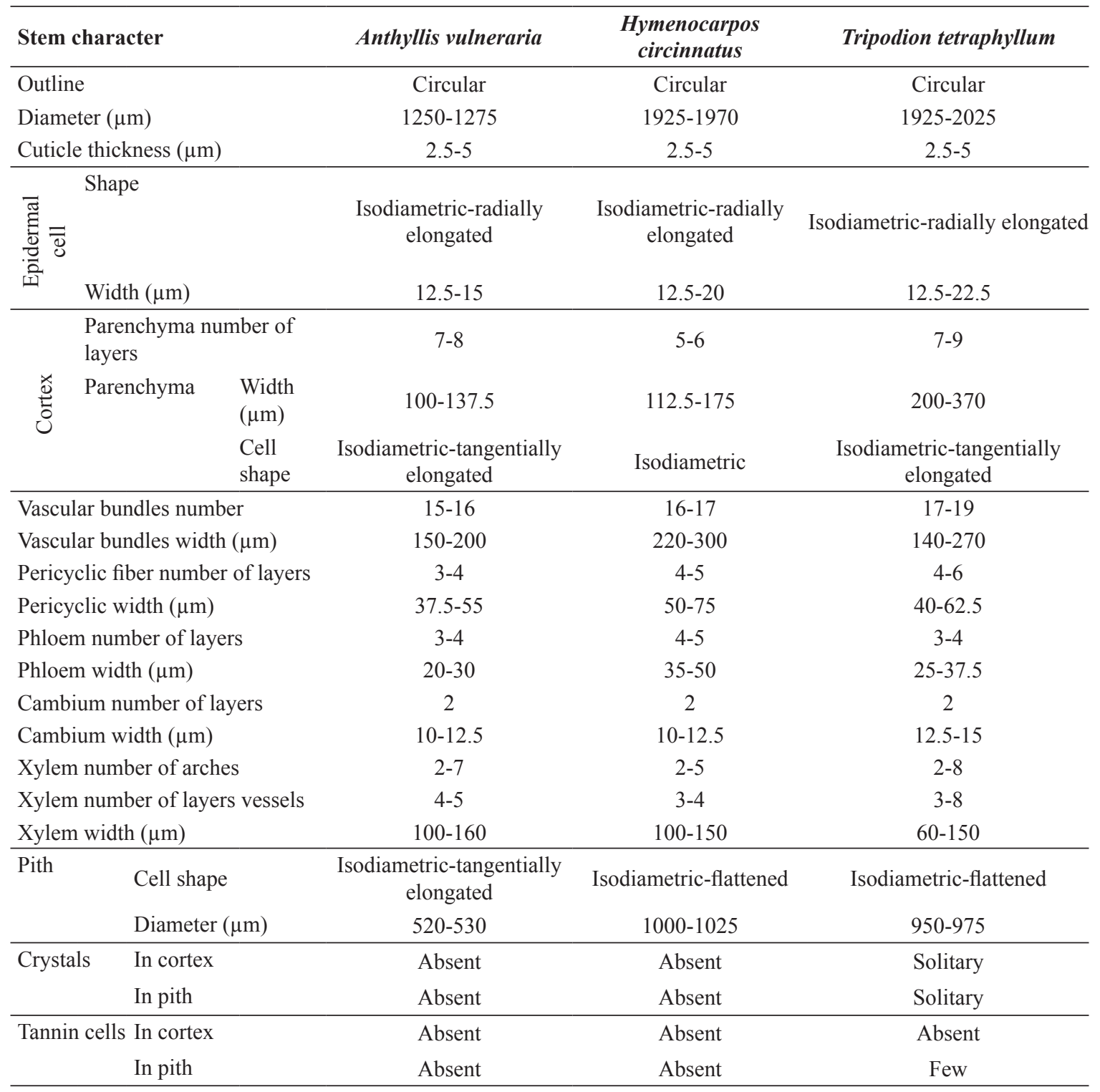

Hymenocarpos circinnatus

The leaf in midrib region is u-shaped, 450$500 \mu \mathrm{m}$ thickness, upper and lower epidermis uniseriate, $12.5-37.5 \mu \mathrm{m}$ thickness, covered with $2.5 \mu \mathrm{m}$ cuticle thickness. In midrib region, parenchyma 3-4 layers, angular-irregular, 120$132.5 \mu \mathrm{m}$ thickness. Xylem 3-4 arches, 3-5 vessels per arch, xylem arch 87.5-107.5 $\mu \mathrm{m}$ length. Phloem 3-4 layers, 25-30 $\mu$ m thickness. Phloem fibers 3-4 layers, 25-37.5 $\mu \mathrm{m}$ thickness. Below the main vascular bundles 3-4 layers of parenchyma cells, $112.5-125 \mu \mathrm{m}$ thickness. The wings $280-310 \mu \mathrm{m}$ thickness, mesophyll tissue chlorophyllous with wide air spaces, 5-6 rows, $210-275 \mu \mathrm{m}$ thickness.

\section{Tripodion tetraphyllum}

The leaf in midrib region is u-shaped, 480$500 \mu \mathrm{m}$ thickness, Upper and lower epidermis uniseriate, $15-40 \mu \mathrm{m}$ thickness, covered with $2.5 \mu \mathrm{m}$ cuticle thickness. In midrib region, parenchyma 3-4 layers, flattened-tangentially elongated, 112.5$125 \mu \mathrm{m}$ thickness. Xylem 5-6 arches, 2-3 vessels per arch, xylem arch 55-62.5 $\mu \mathrm{m}$ length. Phloem 3-4 layers, 30-32.5 $\mu \mathrm{m}$ thickness. Phloem fibers 2-3 layers, $12.5-25 \mu \mathrm{m}$ thickness. Below the main vascular bundles 4-5 layers of parenchyma cells, $187.5-200 \mu \mathrm{m}$ thickness, have 2-3 tannin cells. The wings 220-230 $\mu \mathrm{m}$ thickness Mesophyll tissue consists of palisade and spongy tissues. Palisade tissue 2-3 rows, $62.5-85 \mu \mathrm{m}$ thickness, spongy tissue 3-4 rows, $70-87.5 \mu \mathrm{m}$ thickness. 
TABLE 4. Anatomical characters of leaf in the studied taxa.

\begin{tabular}{|c|c|c|c|c|c|}
\hline \multicolumn{3}{|c|}{ Character } & Anthyllis vulneraria & $\begin{array}{l}\text { Hymenocarpos } \\
\text { circinnatus }\end{array}$ & Tripodion tetraphyllum \\
\hline \multicolumn{3}{|c|}{ Shape } & $\mathrm{V}$ & $\mathrm{U}$ & $\mathrm{U}$ \\
\hline \multicolumn{3}{|c|}{ Midrib thickness $(\mu \mathrm{m})$} & $400-420$ & $450-500$ & $480-500$ \\
\hline \multicolumn{3}{|c|}{ Cuticle thickness $(\mu \mathrm{m})$} & 2.5 & 2.5 & 2.5 \\
\hline \multirow{2}{*}{\multicolumn{2}{|c|}{ Epidermal cells }} & Thickness $(\mu \mathrm{m})$ & $\begin{array}{l}12.5-25 \text { up. } \\
12.5-20 \text { lo. }\end{array}$ & $\begin{array}{l}\text { 20-37.5 up. } \\
12.5-22.5 \text { lo. }\end{array}$ & $\begin{array}{l}25-40 \text { up. } \\
15-20 \text { lo. }\end{array}$ \\
\hline & & Shape & $\begin{array}{l}\text { Isodiametric- } \\
\text { tangentially elongated }\end{array}$ & $\begin{array}{l}\text { Tubular- } \\
\text { tangentially } \\
\text { elongated }\end{array}$ & $\begin{array}{c}\text { Isodiametric-tangentially } \\
\text { elongated }\end{array}$ \\
\hline \multirow{13}{*}{ 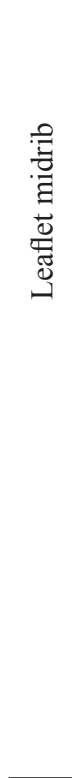 } & \multicolumn{2}{|l|}{ Xylem number of arches } & $4-6$ & $3-4$ & $5-6$ \\
\hline & \multicolumn{2}{|l|}{ Xylem thickness $(\mu \mathrm{m})$} & $75-82.5$ & $87.5-107.5$ & $55-62.5$ \\
\hline & \multicolumn{2}{|c|}{ Xylem number of vessels in arch } & $2-6$ & $3-5$ & $2-3$ \\
\hline & \multicolumn{2}{|l|}{ Phloem number of layers } & $3-4$ & $3-4$ & $3-4$ \\
\hline & \multicolumn{2}{|l|}{ Phloem thickness $(\mu \mathrm{m})$} & $20-25$ & $25-30$ & $30-32.5$ \\
\hline & \multicolumn{2}{|l|}{ Parenchyma number of layers } & $\begin{array}{l}2-3 \text { up. } \\
3-4 \text { lo. }\end{array}$ & $\begin{array}{l}3-4 \text { up. } \\
3-4 \text { lo. }\end{array}$ & $\begin{array}{l}3-4 \text { up. } \\
4-5 \text { lo. }\end{array}$ \\
\hline & \multirow[t]{2}{*}{ Parenchyma } & Thickness $(\mu \mathrm{m})$ & $\begin{array}{l}\text { 87.5-100 up. } \\
100-107.5 \text { lo. }\end{array}$ & $\begin{array}{l}120-132.5 \text { up. } \\
112.5-125 \text { lo. }\end{array}$ & $\begin{array}{l}\text { 112.5-125 up. } \\
187.5-200 \text { lo. }\end{array}$ \\
\hline & & Cell shape & Isodiametric- flattened & Angular-irregular & $\begin{array}{l}\text { Flattened-tangentially } \\
\text { elongated }\end{array}$ \\
\hline & & Absent & Absent & Absent \\
\hline & \multicolumn{2}{|l|}{ fiber number of layers } & $3-4$ & $3-4$ & $2-3$ \\
\hline & \multicolumn{2}{|l|}{ Fiber thickness $(\mu \mathrm{m})$} & $25-37.5$ & $25-37.5$ & $12.5-25$ \\
\hline & \multirow[t]{2}{*}{ Tannin cells } & $\begin{array}{l}\text { Upper vascular } \\
\text { bundle }\end{array}$ & Absent & Absent & $2-3$ \\
\hline & & $\begin{array}{l}\text { Lower vascular } \\
\text { bundle }\end{array}$ & Absent & Absent & Absent \\
\hline \multirow{9}{*}{ 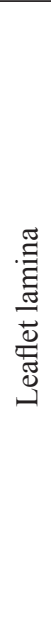 } & \multicolumn{2}{|l|}{ Thickness $(\mu \mathrm{m})$} & $300-350$ & $280-310$ & $220-230$ \\
\hline & \multirow[t]{2}{*}{$\begin{array}{l}\text { Mesophyll with interspace } \\
\text { widely }\end{array}$} & $\begin{array}{l}\text { Number of } \\
\text { rows }\end{array}$ & $5-6$ & $5-6$ & - \\
\hline & & $\begin{array}{l}\text { Thickness } \\
(\mu \mathrm{m})\end{array}$ & $250-315$ & $210-275$ & - \\
\hline & \multirow[t]{2}{*}{ Palisade layer } & $\begin{array}{l}\text { Number of } \\
\text { rows }\end{array}$ & - & - & $2-3$ \\
\hline & & $\begin{array}{l}\text { Thickness } \\
(\mu \mathrm{m})\end{array}$ & - & - & $62.5-85$ \\
\hline & \multirow[t]{2}{*}{ Spongy layer } & $\begin{array}{l}\text { Number of } \\
\text { rows }\end{array}$ & - & - & $3-4$ \\
\hline & & $\begin{array}{l}\text { Thickness } \\
(\mu \mathrm{m})\end{array}$ & - & - & $70-87.5$ \\
\hline & \multicolumn{2}{|l|}{ Tannin cells } & Absent & Absent & Absent \\
\hline & \multicolumn{2}{|l|}{ Crystals } & Solitary & Absent & Absent \\
\hline
\end{tabular}

SEM of the spermoderm (Table 5 and Fig. 4)

SEM of the epidermal cells clarifies the texture and reticulation of their anticlinal (radial) walls, the appearance of the outer periclinal walls and the persistency of the primary cell walls.

Anthyllis vulneraria

Seed ovoid with rounded poles, $2-3 \times 1-1.5 \mathrm{~mm}$ size, brown or yellowish with terminal part pale green in colour, covered with wax, hilum circular, $83.87 \times 80.65 \mu \mathrm{m}$ size, subapical in position, rim aril raised, micropyle obtriangular, and $7.8 \times 7.8 \mu \mathrm{m}$ size . Seed coat pattern irregularly reticulate, anticlinal wall wavy, relief of cell boundary slightly channeled, thickness of cell boundary moderate, curvature of outer periclinal wall smooth and concave. 

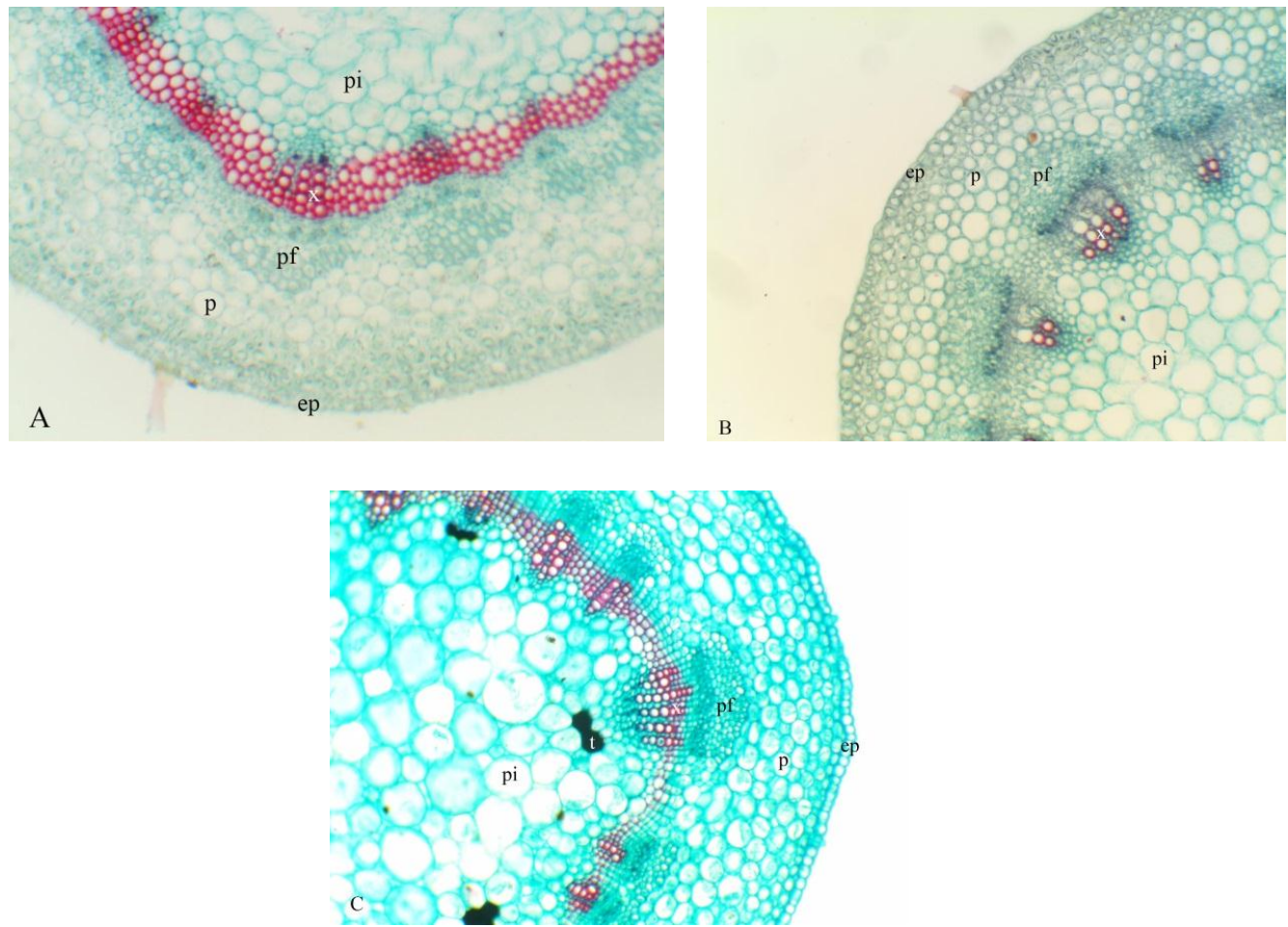

Fig. 2. Stem anatomy of the studied taxa; (A) Anthyllis vulneraria, (B) Hymenocarpos circinnatus, (C) Tripodion tetraphyllum $[\mathrm{ep}=$ Epidermis, $\mathrm{p}=$ Pith, $\mathrm{x}=$ Xylem, $\mathrm{p}=$ Parenchyma, $\mathrm{p}=$ Phloem fiber, $\mathrm{t}=$ Tannin cell $(\mathrm{x}$ 100)] .
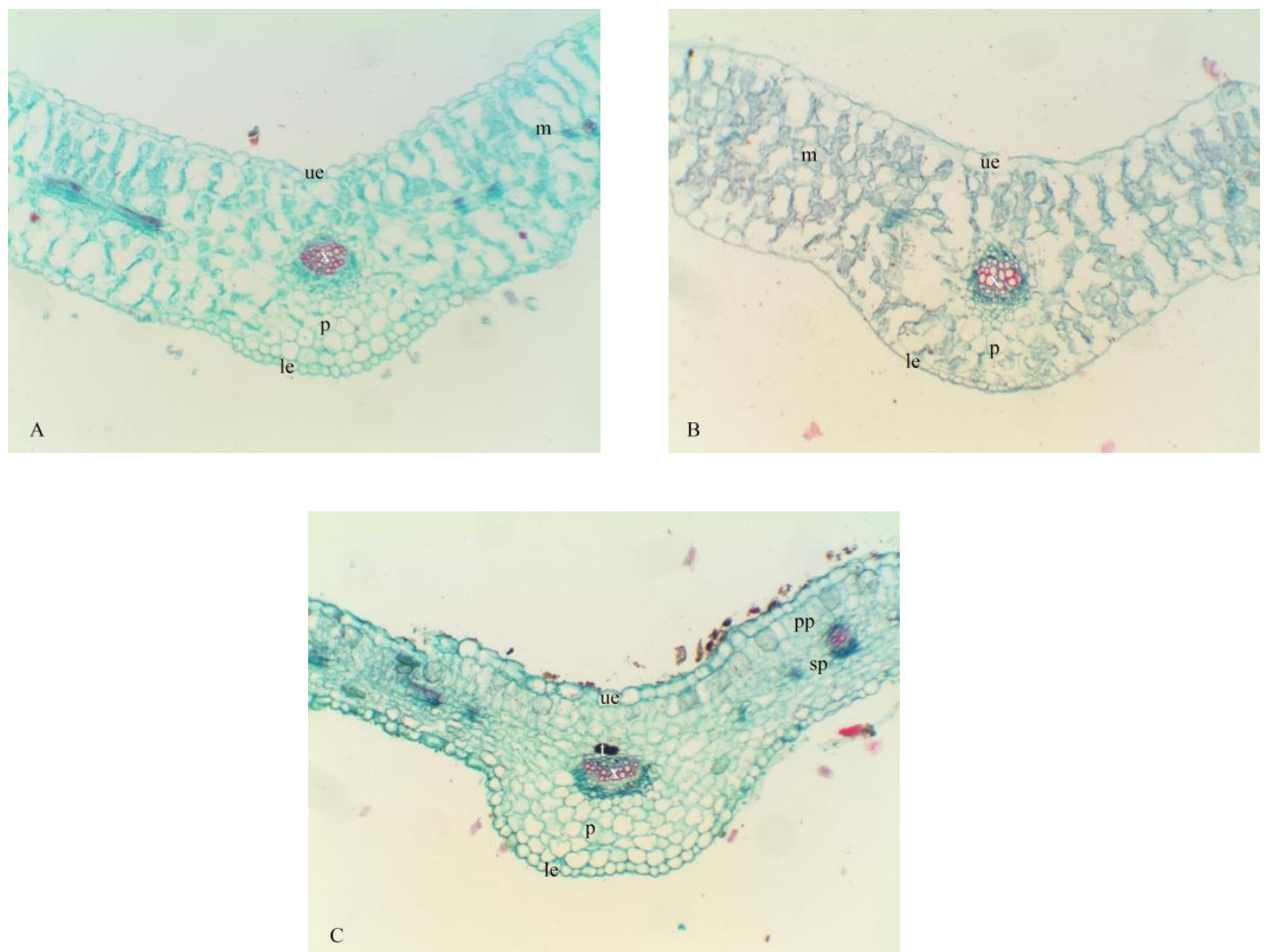

Fig. 3. Leaf anatomy of the studied taxa, (A) Anthyllis vulneraria, (B) Hymenocarpos circinnatus, (C) Tripodion tetraphyllum [ue $=$ Upper epidermis, $\mathbf{x}=$ Xylem, $\mathrm{p}=$ Parenchyma; $\mathbf{m}=$ Mesophyll, $\mathrm{pp}=$ Palisade parenchyma, $\mathbf{s p}=$ Spongy parenchyma, le $=$ Lower epidermis, $t=$ Tannin cell $(x$ 50) $]$. 
TABLE 5. The morphological aspect of the spermoderm of the studied taxa.

\begin{tabular}{|c|c|c|c|c|}
\hline Character & & Anthyllis vulneraria & $\begin{array}{l}\text { Hymenocarpos } \\
\text { circinnatus }\end{array}$ & Tripodion tetraphyllum \\
\hline Seed colour & & $\begin{array}{l}\text { Yellowish with green in } \\
\text { terminal part - brown }\end{array}$ & $\begin{array}{l}\text { Yellowish - creamy } \\
\text { - pale brown, hilum } \\
\text { blackish brown }\end{array}$ & Brown with black spots \\
\hline \multirow{2}{*}{ Seed size } & $\mathrm{L} \times \mathrm{W}(\mathrm{mm})$ & $2-3 \times 1-1.5$ & $3-3.5 \times 2-2.5$ & $3-3.5 \times 2-2.5$ \\
\hline & $\mathrm{L} / \mathrm{W}$ ratio & 2 & 1.4 & 1.4 \\
\hline Seed pole & & Rounded & Rounded & Rounded-truncate \\
\hline Seed shape & & Ovoid & Reniform & $\begin{array}{l}\text { Oblong - Ellipsoid } \\
\text { Crispate }\end{array}$ \\
\hline Seed coat pattern & & Reticulate & Micropappilate in rows & Papillae \\
\hline \multirow{3}{*}{ Hilum } & Position & Subapical & Lateral & Lateral \\
\hline & Shape & Circular & Oblate & Ovate \\
\hline & $\mathrm{L} \times \mathrm{W}(\mu \mathrm{m})$ & $83.87 \times 80.65$ & $75.81 \times 88.71$ & $83.87 \times 77.42$ \\
\hline Rim aril & & Raised & Raised & Raised \\
\hline \multirow{2}{*}{ Micropyle } & Shape & Obtriangular & Narrow oblong & $\begin{array}{c}\text { Narrow oblong with one } \\
\text { open end }\end{array}$ \\
\hline & $\mathrm{L} \times \mathrm{W}(\mu \mathrm{m})$ & $7.8 \times 7.8$ & $7.8 \times 1.56$ & $9.36 \times 1.56$ \\
\hline \multicolumn{2}{|l|}{ Outline of cells } & Reticulate-irregular & Oblong-radial elongated & Tetra-hexagonal \\
\hline \multicolumn{2}{|l|}{ Anticlinal wall } & Wavy & Wavy & Lobbed \\
\hline \multicolumn{2}{|l|}{ Relief of cell boundary } & Slightly channeled & Slightly channeled & Channeled \\
\hline \multicolumn{2}{|l|}{ Thickness of cell boundary } & Moderately & Moderately & Very thick \\
\hline \multicolumn{2}{|c|}{ Curvature of outer periclinal wall } & Concave-smooth surface & Concave-smooth surface & Concave- irregular \\
\hline \multicolumn{2}{|l|}{ Wax } & Present & Present & Present \\
\hline
\end{tabular}

\section{Hymenocarpos circinnatus}

Seed reniform, 3-3.5 × 2-2.5mm size, yellowishcreamy-pale brown in colour with hilum blackish, seed poles rounded. Seed coat pattern micropapilate in rows, outline of cells oblong-radial elongated, anticlinal wall wavy, relief of cell boundary slightly channeled, thickness of cell boundary moderately, curvature of outer periclinal wall concave, smooth surface. Hilum oblate, $75.81 \times 88.71 \mu \mathrm{m}$ size, lateral in position. Rim aril raised. Micropyle narrow oblong, $7.8 \times 1.56 \mu \mathrm{m}$ size. Wax present.

\section{Tripodion tetraphyllum}

Seed oblong-ellipsoid crispate, $3-3.5 \times 2-2.5 \mathrm{~mm}$ size, brown with black spots in colour, seed poles rounded-truncate. Seed coat pattern papillae in rows, outline of cells tetra-hexagonal, anticlinal wall lobbed, relief of cell boundary channeled, thickness of cell boundary very thick, curvature of outer periclinal wall concave irregular. Hilum ovate, $83.87 \times 77.42 \mu \mathrm{m}$ size, lateral in position. Rim aril raised. Micropyle narrow oblong with one open end, $9.36 \times 1.56 \mu \mathrm{m}$ size. Wax present.

\section{Discussion}

Several attempts have been made in distinguishing and identification of the genera Anthyllis, Hymenocarpos and Tripodion. Taubert (1894) included Hymenocarpos as synonym to Circinas. Sokoloff (2003 a) have treated Hymenocarpos as synonym to Anthyllis (subgenus Cornicina, section Hymenocarpos). Tikhomirov \& Sokoloff (1996), Benedi (2000) and Sokoloff (2003 a) have treated Anthyllis tetraphylla as genus Tripodion.

The morphological characteristics of the studied taxa revealed that they varied from each other and can be distinguished depending on the habit, characteristics of leaves, inflorescence, flowers, pods, and seeds.

The use of anatomical characters in taxonomic investigation is becoming of increasing interest. Anatomical structure is most likely providing evidence helping to establish the affinities of genera of uncertain taxonomic status, yet in the same time proves very helpful for individual identification (Welkie \& Caldweli, 1970 and Osmond et al., 1980). Turki (2007) and Kasem (2016) reported the importance of anatomical differences in the distinction among species. 

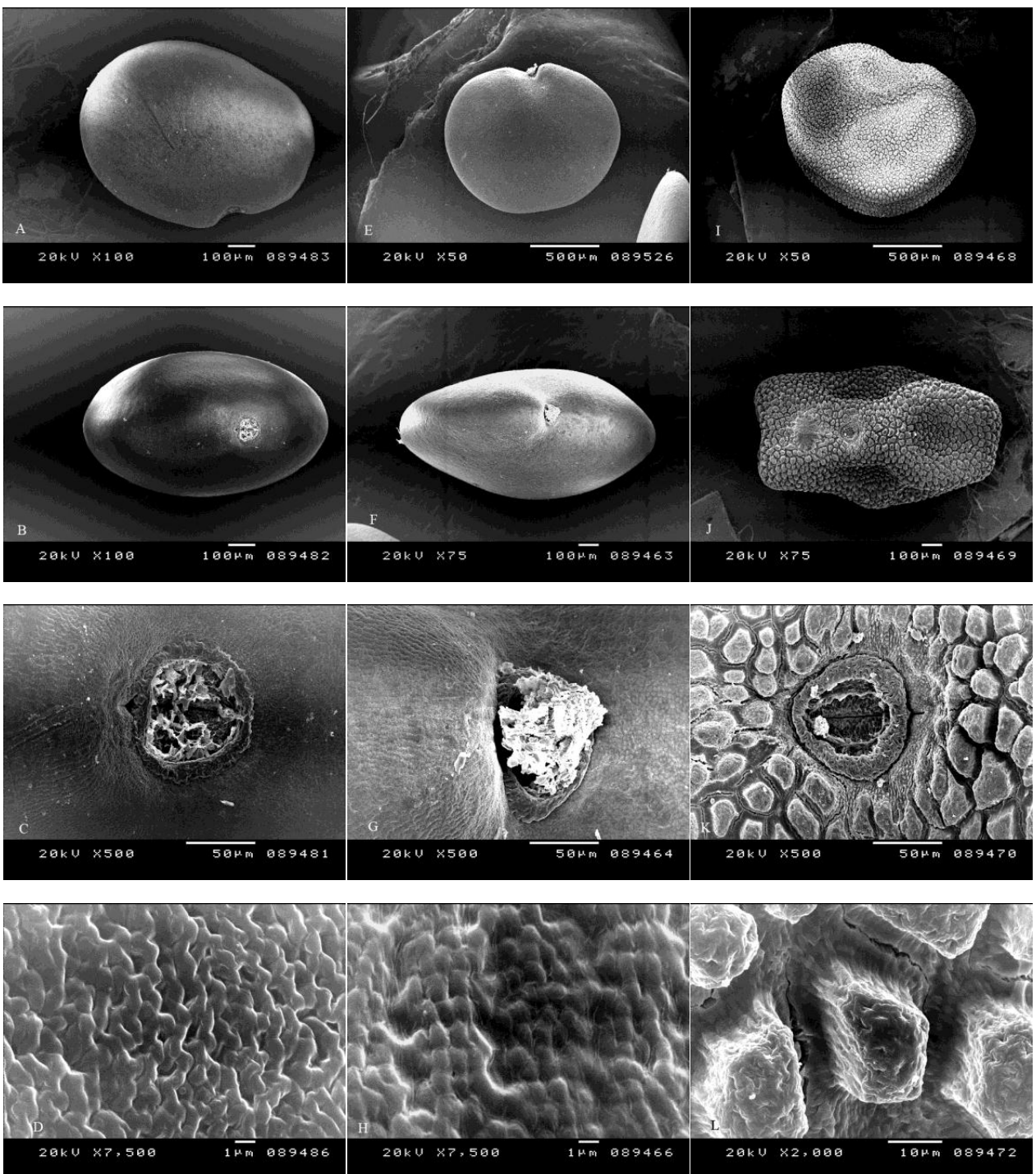

Fig. 4. SEM micrograph of spermoderm surface of the studied taxa; (A-D) Anthyllis vulneraria, (E-H) Hymenocarpos circinnatus, (I-L) Tripodion tetraphyllum [A, E, I: Seed morphology; B, F, J: Hilum position; C, G, K: Hilum shape; D, H, L: Spermoderm surface].

The studied taxa displayed remarkable differences in the anatomical investigations of their stems and leaflets. Comparison of the internal structure of the stems revealed differences in cortex, number of vascular bundles, xylem, pith diameter, crystals and tannin cells. Comparison of the internal structure of the leaflets revealed differences in shape and thickness of midrib and xylem, lamina thickness, and crystals and tannin cells.

Anticlinal undulations and characters of cell boundaries in the seed exine are of high taxonomic significance and often characterize between the species and genus level (Barthlott \& Voit, 1979 and Barthlott, 1981). Kaplan et al. (2007) and
Fawzi et al. (2010) reported seed coat characters are successfully employed in the identification and classification of taxa.

The results of spermoderm SEM revealed differences in seed coat pattern, outline cells, relief and thickness of cell boundary, curvature of outer periclinal wall.

The present study, depending on the results of macromorphological and anatomical investigations, SEM studies on seeds clearly indicates considerably differences between the three studied genera Anthyllis, Hymenocarpos and Tripodion and support the treatment of them as different genera. The results agree with Medikus 
(1787) in that Tripodion treated as a separate genus.

Key to the genera

1- Perennial herb, inflorescence head, bract palmatisect, the leaf in midrib region is v-shaped, hilum subapical in position, seed coat pattern irregularly reticulate.. Anthyllis

- Annual herb, inflorescence umbellate or cluster, bract simple, the leaf in midrib region is u-shaped, hilum lateral in position, seed coat pattern otherwise . .2

2- Flower 6-7mm long, calyx campanulate, pod orbicular-reniform, cortex up to 6 layers, seed coat pattern micropapilate......Hymenocarpos

- Flower 14-18mm long, calyx tubular, pod ellipsoid- cylindrical, cortex 7-9 layers, seed coat pattern papillae.

Tripodion

\section{References}

Abd El-Gawad, M.A., Salem, M.O. and Hegazi, A.M. (1989) Anatomy of Alfalfa leaflets as affected by NPK-fertilization and saline irrigation. Ann. Agric. Sci. Moshtohor, 27(3), 1439-1447.

Abd El-Rahman, A.A., Ibrahim, A.A. and Hassan, H.T. (1976) Contribution to the anatomical characters of some xerophytes. Bull. Fac. Sci. Cairo Uni. 49, 139-162.

Akulova, Z.V. (1985) To the question on ways of morphological evolution in the genus Anthyllis L. In: Anonymous (Ed.), Proceedings of $7^{\text {th }}$ Conference of Young Scientists of V.L. Komarov Botanical Institute. VINITI, Moscow, pp. 29-36.

Akulova, Z.V. (1986) The Genus Anthyllis L. in the Flora of USSR: Systematics, Morphology, and Usage. Ph.D. Thesis, Leningrad (in Russian).

Barthlott, W. (1981) Epidermal and seed surface characters of plants. Systematic applicability and some evolutionary aspect. Nord. J. Bot. 1, 345-355.

Barthlott, W. and Voit, G. (1979) Mikromorphologie der Samenschalen und Taxonomie der Cactaceae: Ein rasterelectronen-mikroskopischer uberblick. Plant Syst. Evol. 132, 205-229.

Benedi, C. (2000) Anthyllis, Hymenocarpos. In: "Flora
Iberica" Talavera, S. et al. (Eds.), pp. 829-862 and 868-873. 7(2). Real Jardin Botanico, Madrid.

Boissier, P.É. (1838) "Elenchus Plantarum Novarum...", Genéve.

Boissier, P.É. (1839-1845) "Voyage Botanique dans le Midi de l'Espagne". Vol. 2: 158-164. Paris.

Boissier, P.É. (1872) "Flora Orientalis". Vol. 2:156158.Basilea, Genéve, Lyon.

Boulos, L. (1995) "Flora of Egypt Checklist". AlHadara Publishing, Cairo.

Boulos，L. (1999) "Flora of Egypt". Vol. 1.Al-Hadara Publishing, Cairo.

Boulos, L. (2009) "Flora of Egypt Checklist", Revised annotated edition. Al-Hadara Publishing, Cairo.

Brochmann, C. (1992) Pollen and seed anatomy of Nordic Draba (Brassicaceae) phytogenetic and ecological implications. Nordic J. Botany, 12(6), 657-673.

Castroviejo, S. (ed.) (2000) "Flora Iberica". Vol VII (II) Leguminosae (Partim). Real Jardín Botánico (CSIC), Madrid, Spain.

El-Hadidi, M.N. and Fayed, A.A. (1994/95) Material for Excursion Flora of Egypt - Taeckholmia, 15, $1-223$.

Fawzi, N.M., Fawzy A.M. and Mohamed, A.A.H.A. (2010) Seed morphological studies on some species of Silene L. (Caryophyllaceae). Inter. J. Bot. 6(3), 287-292.

Gussone, G. (1827/28) "Florae Siculae Prodromus". Regia Typographia. Neapoli.

Kaplan, A., Hasanoğlu, A. and Ince, I.A. (2007) Morphological, anatomical and palynological properties of some Turkish Veronica L. species (Scrphulariaceae). Inter. J. Bot. 3(1), 23-32.

Kasem, W. (2016) Anatomical, pollen grains and seed exomorphic studies on five species of Cleome L (Cleomaceae Bercht. \& Presl) collected from South West of Saudi Arabia. J. Plant Sciences, 4, 29-36.

Kirkbride, J.H., Gunn, C.R. and Weitzman, A.L. (2003) Fruits and seeds of genera in the subfamily 
Faboideae (Fabaceae). United States Department of Agriculture, Technical Bulletin 1208 No. 1890.

Lassen, P. (1986) In: "Hymenocarpos and Tripodion". W. Greuter and T. Raus (Eds.), 16, pp. 111-112. Med-Checklist notulae, 13. Willdenowia.

Lassen, P. (1987) In: W. Greuter \& T. Raus (eds.), MedChecklist notulae, 14. Willdenowia 16: 443.

Lersten, N.R. (1981) Testa topography in Leguminoseae subfamily Papilionoideae. Proc. Iowa Acad. Sci. 88(4), 180-191.

Linnaeus, C. (1753) Species plantarum, Vol II. Stockholm, Sweden.

Medikus, F.K. (1787) Neue methode bei Pflanzen $\mathrm{zu}$ ordnen. Vorlesesungen der Churpf lzischen physicalisch-conomischen Gesellschaft, 2, 354382.

Moench, C. (1794) A staminum situ describendi. Methodus Plantas Horti Botanici et Agri Marburgensis, 1, 1-368.

Osmond, C.B., Bjorkman and Anderson, D.J. (1980) Physiological process in plant Ecology towards a synthesis with Atriplex. - Berlin.

Pandey, B.P. (1982) "Plant Anatomy". New Delhi.

Polhill, R.M. (1981) Papilionoideae. In: " Advances Legume Systematics", R.M. Polhill and P.H. Raven (Eds.), part 1, pp. 191-208. Royal Botanic Gardens, Kew.

Sass, J.E. (1961) "Botanical Microtechnique", $3^{\text {rd }}$ ed. Amsterdam.

Savi, G. (1798) "Flora Pisana", Vol. 1-2. Pietro
Giacomelli Editore, Pisa.

Sokoloff, D.D. (2003 a) On system and phylogeny of the tribe Loteae DC. (Leguminosae) [in Russian]. Bulletin of Moscow Society of Naturalists, Biological Series, 108(3), 35-48.

Sokoloff, D.D. (2003 b) Morphology and Classification of the Tribe Loteae DC. of the Family Leguminosae. Ph.D. Thesis, Moscow (in Russian).

Stearn, W.T. (1992) "Botanical Latin", $4^{\text {th }}$ ed. Timber Press, Oregon, USA.

Täckholm, V. (1974) "Students' Flora of Egypt". $2^{\text {nd }}$ ed., Cairo University, Egypt.

Taubert, P. (1894) In: "Leguminosae". Engler, A., Prantl, K., Die naturlichen Pflanzenfamilien 3:W. Engelmann, Leipzig, pp. 70-385.

Tikhomirov, V.N. and Sokoloff, D.D. (1996) On the division of the genus Anthyllis L. (Papilionaceae, Loteae) into subgenera and sections. Bulletin of Moscow Society of Natura;ists, Biological Series, 101, part 1, 61-73 (in Russian).

Tournefort, J. (1719) Institutione rei herbariae, 3rd ed., Paris.

Turki,Z.A. (2007) The genus Ammannia L. (Lythraceae) in Egypt. Flora Mediterranea, 17, 97-114.

Welkie, J.T. and Caldweli, M. (1970) Leaf anatomy of species in some dicotyledon. Families as related to the $\mathrm{C} 3$ and $\mathrm{C} 4$ pathways of Carbon fixation. Canad. J. Bot. 48, 2135-2146.

\section{دراسات تصنيفية لعشيرة Loteae (الفصيلة الفولية) في مصر. تحت العشيرة Tripodion) Anthyllidinae و (Anthyllis, Hymenocarpos

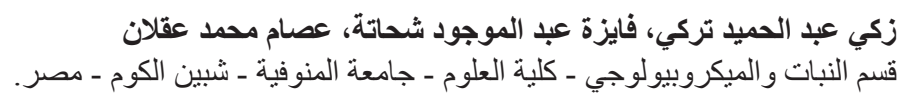

تهدف الدراسة إعادة تقييم العلاقات التصنيفية المتداخلة بين أجناس Hymenocarpos ، Anthyllis و الظئس

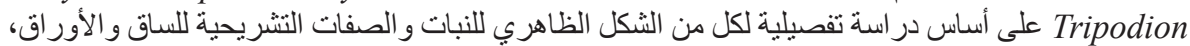

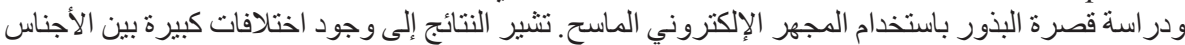

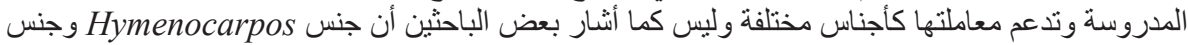

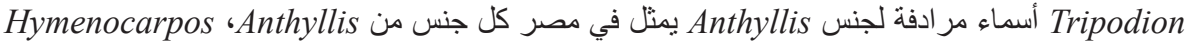

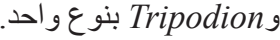

